\title{
The Globular Cluster Specific Frequency in NGC 1399 and 4486: A Comparative Study
}

\author{
Juan C. Forte \\ Fac. Cs. Astronómicas y Geofísicas, Univ. Nac. de La Plata, Paseo del \\ Bosque, La Plata 1900, and CONICET, Argentina \\ Doug Geisler \\ Grupo de Astronomía, Dpto. de Física, Univ. de Concepción, Casilla \\ 160-C, Concepción, Chile \\ E. Kim, Myung Gyoon Lee \\ Astronomy Program, Seoul National Unversity, Shinlim-dong, \\ Gwanag-gu, Seoul 151-542, South Korea \\ Pablo Ostrov \\ Fac. Cs. Astronómicas y Geofísicas, Univ. Nac. de La Plata, Paseo del \\ Bosque, La Plata 1900, Argentina
}

\begin{abstract}
A two color CCD survey for globular clusters in the galactocentric range from 2 to 7 arcmin from the galaxy centers is presented for NGC 1399 and NGC 4486 (M87), two systems that, for a long time, have been considered as class archetypes of the so called "high specific frequency phenomenon". The new results, combined with previously published HST data for the inner 2 arcmin, and with a re-discussion of the surface brightness profiles, allow a new estimate of the globular cluster specific frequencies. The resulting $S_{N}$ values cannot be considered as anomalously large and, rather, they are consistent with values obtained for other galaxies with similar morphologies.
\end{abstract}

\section{Introduction}

The fact that some galaxies exhibit large values of the globular cluster specific frequency $S_{N}$ (Harris \& van den Bergh 1981) has originated a number of interesting astrophysical scenarios aiming at explaining this situation. Among these galaxies, NGC 4486 at the center of the Virgo cluster, and NGC 1399 in a similar situation in Fornax, have been considered for a long time as archetypes. The estimates of $S_{N}$ in these galaxies have been based on ground telescope observations and using single color photometric surveys (e.g. McLaughlin, Harris \& Harris 1994), i.e., non-resolved object counts around the galaxies were corrected from the effects of field interlopers by subtracting the non-resolved object counts observed in a comparison and within the same magnitude range. area. This approach, however, suffers from the high statistical noise associated with the steep 
luminosity function of field objects that, in turn, led to large uncertainties in the determination of the position of the turn-over of the integrated globular cluster luminosity function. The use of two color surveys (as presented in this work) alleviates the problem since globular candidates are looked for within a given color range (e.g. that covered by MW globulars) then decreasing the contamination level. On the other side, reliable determinations of the turn overs, based on HST observations, are now available for the inner regions of both galaxies (Kundu et al. 1999; Grillmair et al. 1999).

Another source of uncertainty that has an impact on the $S_{N}$ values comes from the adopted total galaxy brightness within a given galactocentric radius. For example, the use of extrapolated magnitudes derived from aperture photometry of the central regions may lead, in the case of $\mathrm{cD}$ galaxies, to a large underestimation of the total brightness (see, for example, Burstein et al. 1987).

\section{Observations and Data Handling}

Observations were made with 2048 pixels on a side CCDs that, with the CTIO and KPNO $4 \mathrm{~m}$ telescopes, gave an areal coverage of some $200 \mathrm{sq}$. '. The images were taken with the $\mathrm{C}$ and $T_{1}$ filters of the Washington photometric system in very good seeing conditions. Image classification and photometry, as well as completeness experiments, were carried out using the ALLFRAME version of the DAOPHOT package (Stetson 1987). The analysis described in the following sections is restricted to the galactocentric region defined between 2 and $7^{\prime}$. In the case of NGC 1399 the areal completeness, in that region, is about $90 \%$ and $65 \%$ for NGC 4486.

Surface brightness (B and R) profiles were obtained for NGC 1399 by means of a $2.1 \mathrm{~m}$ telescope (CASLEO, Argentina) and using off set frames and an airglow sensor in order to estimate the sky level (Faifer, Forte \& Pellizza in preparation).

\section{The Globular Cluster Populations}

As a working definition we considered as a globular cluster candidate all the non-resolved objects within a magnitude range from $T_{1}=18$ to $T_{1}=24$ (the limiting magnitude of the survey) and $\left(C-T_{1}\right)$ colors from 0.9 to 2.1 . This is the color range spanned by MW globulars. The statistics within that domain were corrected from field contamination. The level of this contamination was derived using an iterative approach (McLoughlin et al. 1994) for NGC 4486, and a 78 sq. 'comparison field for NGC 1399. This field is located some 40 'from the center of the galaxy (Forte et al. 2001). To within the uncertainties, the areal density and color distributions of field interlopers resulted very similar in both cases.

A smoothed color distribution was obtained for each GC population by convolving the observed $\left(C-T_{1}\right)$ colors with a gaussian kernel $(0.07$ mags. dispersion) and, after removing the field contamination, are depicted in Figure 1. This figure includes all GCs candidates down to $T_{1}=23.2$ (the approximate position of the luminosity function turn-overs) and has a completeness above 90 \%. In both GCs systems the color distribution is clearly bimodal. 
Azimuthal counts were performed on both GCs samples aiming at obtaining the ellipticity of the systems and to determine their boundaries for a comparison with the underlying galaxy halos. For the NGC 4486 GCs we obtain $\epsilon=0.24 \pm$ 0.02 , very similar to that of the halo (see, for example, fig. 15 in McLoughlin et al. 1994). In turn, the NGC 1399 globulars, with $\epsilon=0.10 \pm 0.05$, exhibit a spatial distribution that seems slightly rounder than the galaxy light.

\section{Galaxy Surface Brightness}

A composite visual surface brightness profile was obtained for NGC 4486 by combining the CCD photometry given by Boroson, Thompson \& Schectman (1983) and the photographic photometry obtained by Carter and Dixon (1978). Simulated aperture photometry on the first of these profiles shows excellent agreement with aperture photometry from Burstein et al. (1987). In turn, a comparison between the profiles shows that the Carter and Dixon one requires a zero point correction of -0.14 mags. It is worth noting that, the de Vaucouleus \& Nieto (1978) east-west blue scan, after a correction that takes into account both the orientation and ellipticity of the NGC 4486 halo, shows a good agreement with the Carter \& Dixon profile (adopting $(B-V)=1.0$ ).

The visual profile for NGC 1399 was derived from B and R photometry (Faifer et al. in preparation) and using a $(V-R)$ vs. $(B-R)$ color relation similar those discussed in Forbes \& Forte (2001). This profile, on the other side, shows a good overall agreement with the photographic profile obtained by Schombert (1986), exhibiting deviations of, at most, 0.1 mags.

The integration of the described profiles up to an elliptical galactocentric radius of 7 'yields $V=8.54 \pm 0.10$ and $V=9.02 \pm 0.06$ for NGC 4486 and NGC 1399 , respectively.

\section{The Globular Cluster Specific Frequencies}

Figure 2 displays the integrated luminosity functions for clusters with elliptical radii from 2 to 7 'in NGC 4486 and NGC 1399 (corrected for completeness and field contamination). The gaussian fits show that, within the uncertainties, both systems have very similar turn-overs at $T_{1}=23.2 \pm 0.10$. However, the NGC 4486 system exhibits a dispersion of 1.5 mags. that is considerably larger than in NGC 1399, with 1.25 mags. The cluster population in both galaxies results $4700 \pm 400$ and $2300 \pm 300$ respectively. A preliminary analysis of the luminosity function for red and blue clusters shows that the former ones seem to have fainter turn-overs (by 0.2 to 0.3 mags).

The GC populations within a 2 'galactocentric radius were estimated from the HST density profiles given by Kundu et al. (1999) for NGC $4486(1100 \pm 200$ clusters) and Forbes et al. (1998) for NGC 1399 (700 \pm 150 clusters). Finally, a combination of these results yield a total GC population of $5800 \pm 450$ for NGC 4486 and $3300 \pm 350$ for NGC 1399 (within an elliptical galactocentric radius of 7 arcmin).

For the estimate of the $S_{N}$ parameter, we adopted a distance modulus of $(V o-M v)=31.0$ for the Virgo cluster (Graham et al. 1999) and $(V o-M v)=$ 31.3 for the Fornax cluster (Prosser et al. 1999). These distance moduli imply 

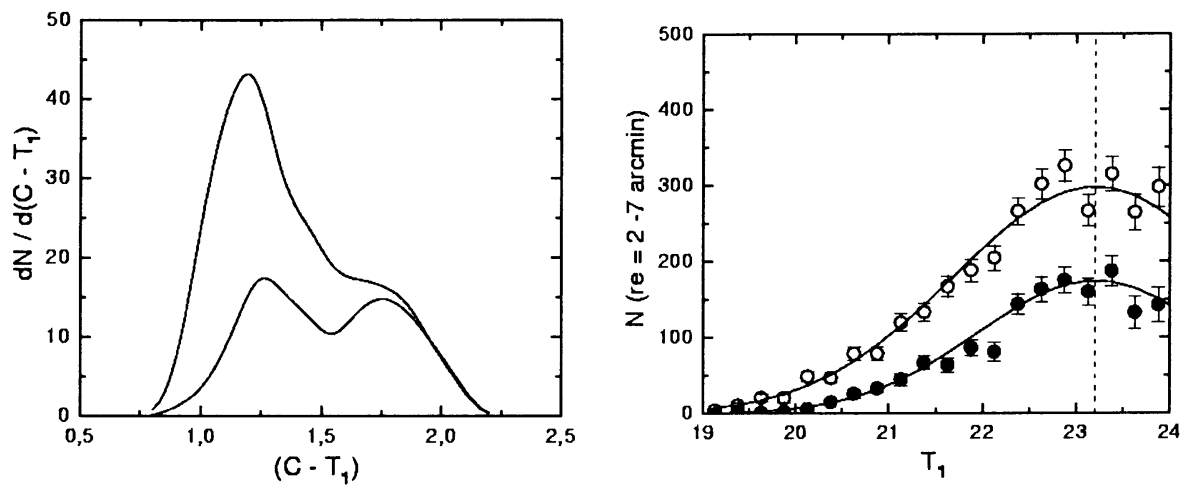

Figure 1. Left panel: Generalized color histograms for GCs in NGC 4486 (upper curve) and NGC 1399. Right panel: Integrated luminosity functions for GCs in NGC 4486 (upper curve) and NGC 1399. The dashed vertical line is at $T_{1}=23.2$. Both panels correspond to clusters with elliptical galactocentric radii from 2 to $7^{\prime}$.

$S_{N}=6 \pm 1$ for NGC 4486 and $S_{N}=3.7 \pm 0.8$ for NGC 1399. This last value would increase to $S_{N}=5.3$ if $(V o-M v)=31.0$ is adopted (i.e. if both galaxies are at the same distance as suggested by the similarity of the turn-over magnitudes shown in Figure 2).

\section{Conclusions}

The $S_{N}$ values obtained in this work both for NGC 4486 and 1399 are considerably lower than previous estimates given in the literature. A first result in this direction was in fact reported in Ostrov, Forte \& Geisler (1998) for NGC 1399 although the photometry in that paper had a smaller areal coverage and a brighter limiting magnitude.

The new specific frequencies are comparable with values observed in other central galaxies in clusters and cannot be considered as anomalously large. For example, figure 13 in Harris et al. (1998) shows that, previous estimates of $S_{N}$ in both galaxies led to highly deviant positions in the $S_{N}$ vs. galaxy velocity dispersions (within the cluster) relations. This discrepancy is removed when using the $S_{N}$ values presented in this work.

An analysis of the literature strongly suggests that spuriously large values of $S_{N}$ were obtained as a consequence of a combination of uncertainties in the position of the luminosity function turn-overs, leading to an over estimate of the cluster populations and, on the other side, from an under estimation of the total galaxy brightnesses.

Finally, we note that the spatial distribution of the red clusters seems very similar to that of the galaxy halos (and with an approximately similar specific frequencies). The difference in the global $S_{N}$ value is, then, connected with the different abundance of blue globulars. 
Acknowledgments. This work was partially supported with grants from CONICET and the ANPC\&T, Argentina.

\section{References}

Boroson, T.A., Thompson, I.B., Schectman, S.A. 1983, AJ, 88, 1707

Burstein, D. et al. 1987, ApJS, 64, 601

Carter, D., \& Dixon, K. L., 1978, AJ, 83, 6

de Vaucouleurs, G., \& Nieto, J.-L. 1978, ApJ, 220, 449

Forbes, D.A., Grillmair, C.J., Williger, G.M., Elson, R.A.W., Brodie, J.P. 1998, MNRAS, 293, 325

Forbes, D., \&Forte, J.C. 2001, MNRAS, 322, 25

Forte, J.C., Geisler, D., Ostrov, P., Piatti, A., \& Gieren 2001, AJ, 121, 1992

Graham, J. et al. 1999, ApJ, 516, 626

Grillmair, C. J., Forbes, D.A., Brodie, J.P., Elson, R.A.W., AJ, 117, 167

Harris, W.E., Harris, G.L.H., McLaughlin, D.E 1998 ApJ, 115, 180

Harris, W.E., \& van den Bergh, S. 1981, AJ86, 1627

Kundu, A., Whitmore, B.C., Saprks, W.B., Macchetto, F.D., Zepf, S.E., Ashman, K.M. 1999, ApJ, 513, 733

McLaughlin, D.E., Harris, W.E., \& Hanes, D.A. 1994, ApJ, 422, 486

Ostrov, P., Forte, J.C., \& Geisler, D. 1998, AJ, 116, 2854

Prosser, Ch. et al. 1999, ApJ, 525, 80

Schombert, J.M. 1986, ApJS60, 603

Stetson, P. 1987, PASP, 99, 191

\section{Discussion}

T. Bridges: Why are the specific frequencies for M87 and NGC 1399 so much lower than found previously? What are the main factors driving this decrease?

$J$. Forte: Ground based observations led to a very faint turnover (0.5 mag fainter) compared with more recent HST measures. The luminosity of both galaxies was underestimated as well.

C. Grillmair: It is difficult to estimate the luminosity of CD galaxies using light profiles in the outer regions as profiles are very uncertain at only a few percent of the background. Would it be better to use a "local specific frequency" using only the galaxy light in the same annulus as the globular clusters?

J. Forte: Yes, that might be a better approach.

P. Barmby: I think the answer to Carl's question is that yes, people have tried to compute local specific fraquencies - Dean McLaughlin did this in one of his papers (Bill Harris confirms this). 